\title{
Breast Cancer Prognostic Stage
}

National Cancer Institute

\section{Source}

National Cancer Institute. Breast Cancer Prognostic Stage. NCI Thesaurus. Code C139446.

Breast cancer staging used in countries in which biomarker tests are routinely performed. 